\title{
Tailoring the Elastic Postbuckling Response of Cylindrical Shells: A ROUTE FOR EXPLOITING INSTABILITIES IN MATERIALS AND MECHANICAL SYSTEMS
}

\author{
$\mathrm{Nan} \mathrm{Hu}^{1}$, and Rigoberto Burgueño ${ }^{2} *$
}

\begin{abstract}
Postbuckling response, long considered mainly as a failure limit state is gaining increased interest for smart applications, such as energy harvesting, frequency tuning, sensing, actuation, etc. This letter explores the potential of cylindrical shells under axial compression, for which localized buckling events along with high-rate local deformations can be attained in their elastic postbuckling regime under smaller axial system deformation input, as a prototype for harnessing elastic instabilities. Three avenues are presented to tailor and control the elastic postbuckling response of axially-compressed shells: (1) by introducing seeded geometric imperfections (SGI); (2) by introducing non-uniform stiffness distributions (NSD); and (3) by providing lateral constraints and interactions (LCI). Prototyped cylindrical shells were fabricated through 3D printing and tested under loadingunloading cycles. Experimental results show that, with appropriate selection of geometry, material, and stiffness distribution, these three concepts offer significant advantages over uniform cylindrical shells for use of their notoriously unreliable elastic postbuckling response. This work provides new knowledge on the possibilities and means to design the cylindrical shells with controlled elastic postbuckling behavior and opens new avenues for using this structural form for applications in smart materials and structures.
\end{abstract}

\section{KEYWORDS:}

Elastic instability; Cylindrical shells; Postbuckling.

${ }^{1}$ Graduate Student Researcher, hunan2@msu.edu, Department of Civil and Environmental Engineering, Michigan State University, East Lansing, MI, 48824, USA

2 Associate Professor of Structural Engineering, burgueno@msu.edu, Department of Civil and Environmental Engineering, Michigan State University, East Lansing, MI, 48824, USA

* Corresponding Author 


\section{INTRODUCTION}

Elastic instability, long considered mainly as a limit state or a safety guard against ultimate failure, is increasingly gaining a favorable regard. The paradigm shift deals with using the unstable response of flexible and slender structures to design materials and structures with switchable functionalities, reversible reconfigurations, etc. This emerging theme has turned the study of elastic instabilities to a promising research avenue. Harnessing this kind of nonlinear behavior for new purposes was showcased in a recent scientific journal special issue [1]. Major applications include energy harvesting [2], damping [3], sensing [4], actuation [5], predicting material properties [6], morphing structures [7, 8], etc. Elastic instabilities can be found across scales: from nanostructured materials to macro-scale structural components. In a general sense, many interesting problems within this topic remain to be investigated [9], such as new phenomena due to the coupling between geometric and material nonlinearities, and the usefulness of mechanical instabilities for broad engineering applications.

The first step for using a specific unstable event is to identify the desirable features of a given response. Recent research contributions within this avenue have considered elastic instabilities in a variety of structural forms, ranging from one- to three-dimensions, for their opportunity and feasibility to be used as structural prototypes. Examples include the slackening of a twisted thin rod [10], buckling of an elastic planar rod penetrating into a sliding sleeve [11], snapping of an elastic arched beam inspired by a popper [12], snapping of a simple stretched bi-strip made from elastomers [13], the buckling and folding of overcurved rings [14], the shrinking and buckling of thin sheets with non-Euclidean metrics [15, 16], buckling of a planar sheet with a negative-curvature liquid interface [17], the 3D shape of a sheet with a series of prescribed concentric curved folds [18], multi-stability in spontaneous helical ribbons [19], the postbuckling of a thin cylindrical shell under torsional loading to fold 
to a flat 2D form [20], buckling-induced encapsulation of a spherical shell patterned with a regular array of circular voids [21], the secondary buckling instability of an initially spherical elastic capsule [22], etc. These studies have expanded the understanding on mechanical and material instabilities as opportunities rather than modes of failure, and future findings on harnessing elastic instabilities depend on exploring structural prototypes across scales.

The work presented in this letter follows the roadmap of rethinking the classic problem of unstable elastic mechanical response under a new focus. The study focuses on the elastic postbuckling behaviour of cylindrical shells under axial compression, a response so notoriously difficult to predict that is commonly regarded undependable and undesirable. This paper reports on three approaches to tailor the elastic postbuckling response of axially compressed cylindrical shells by: (1) introducing seeded geometric imperfections (SGI); (2) introducing non-uniform stiffness distributions (NSD); and (3) providing internal lateral constraints and interactions (LCI). A prior study [23] by our group has verified the potential avenues of tailoring the postbuckling response of cylindrical shell and this letter presents new numerical and experimental findings on three design concepts with reduced-size shell geometries. Prototyped cylindrical shells were fabricated through 3D printing and tested under axial compression. Discrete maps on response type and response contours on key parameters were numerically established to guide the selection of seeding shape for a target postbuckling response

\section{Postbuckling Response Features and Design Concepts}

The postbuckling response regime of interest in this study is compared to the more traditionally studied regime in Fig. 1(a). Mainstream research efforts have been made since early in the $20^{\text {th }}$ century [24-28] mainly focused on determining the critical buckling load with a single stable equilibrium path, or on determining the initial postbuckling path for use as residual capacity. Yet, the curvature in cylindrical shells provides a natural geometric 
constraint that allows the attainment of multiple elastic equilibrium paths (also known as mode transitions or mode jumps) as the shell transitions into higher order buckling modes at stability critical points. Thus, the aim of this study is on the elastic postbuckling regime and to explore the effect of changing geometry, stiffness distribution and additional constraints on obtaining designable postbuckling response features after the first buckling event.

a

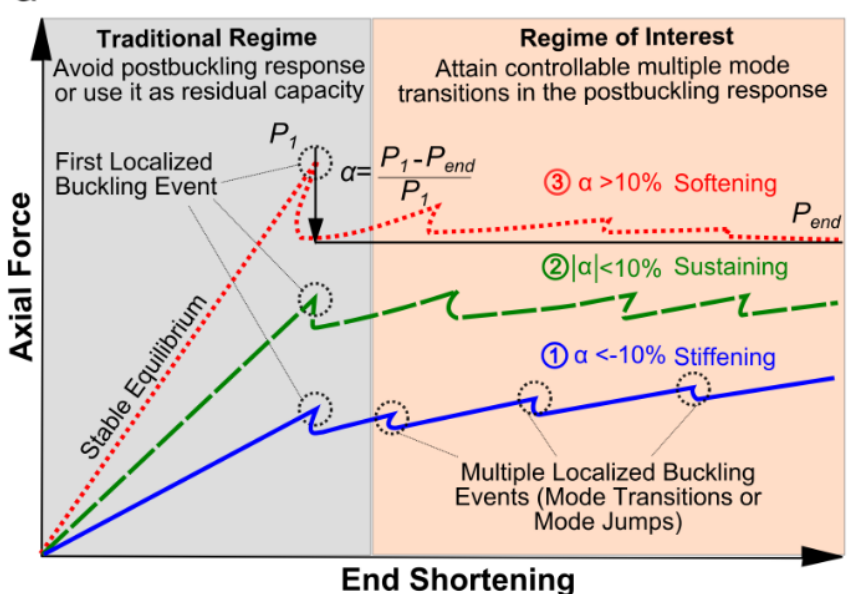

b

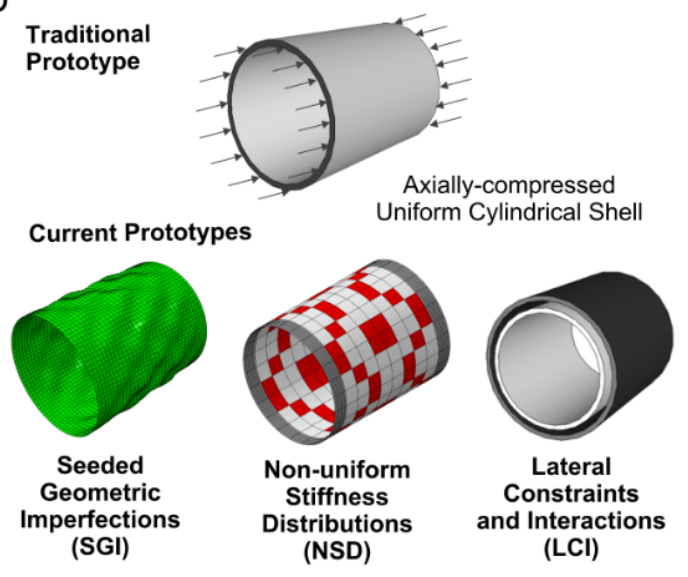

Figure 1. Schematic of the three design concepts on cylindrical shells and target response features.

The number of occurrences of mode transitions and their spacing are important features in the targeted elastic postbuckling response. From a phenomenon point of view, a localized buckling event in the shell surface releases energy from the system and additional strain energy needs to accumulate to generate a new critical event. It is also of interest to notice the hysteretic nature of the response, that is, the enclosed area in the response curve from unloading is associated with the dissipated strain energy from the equilibrium path transitions. Another feature of the postbuckling response is the change in system stiffness after each mode transition and throughout the regime of interest. Based on observed experimental evidence it is proposed that the elastic postbuckling response of axially compressed cylindrical shells can be categorized in three types, as shown in Fig. 1(b): stiffening, sustaining and softening. These definitions are based on a ratio of the total load drop (between the first buckling event and the end of the loading phase) to the magnitude of first critical buckling load. As discussed 
later, each postbuckling response type has its own potential application features. The three specific parameters of interest in the postbuckling response are the total number of mode transitions $\left(n_{t}\right)$, the maximum load drop of a single buckling event $\left(\Delta P_{\max }\right)$, and the enclosed area in the loading-unloading cycle $(A)$. The postbuckling behavior of the cylindrical shells studied in this letter was evaluated under these features and variables.

Three prototypes (SGI, NSD, and LCI) are proposed (see Fig. 1) to achieve a targeted elastic postbuckling response. The SGI concept was inspired by the well-known numerical approach of modeling the postbuckling behavior with the consideration of imperfections. An SGI cylinder is thus obtained by superposing single or multiple mode-shapes from the eigenvalue analyses on a uniform cylinder such that a predefined geometric imperfection provided to the original geometry has a governing role over other initial random imperfections. The NSD cylinder follows a similar concept of introducing artificial imperfections but in this case by strategically placing patterned thickening regions (which alter the stiffness distribution) on the shell surface with a focus of triggering localized buckling events in non-thickened regions. The LCI cylinder concept was driven by the desire to obtain more mode transitions in the postbuckling regime, compared to SGI and NSD cylinders, since the provision of multiple walls leads to their interaction in the elastic postbuckling regime. With the noted variations in geometry, stiffness and boundary conditions, the elastic postbuckling responses of these three cylindrical shell types are expected to be tunable, that is, they can be modified and controlled.

\section{Experimental Methods}

The baseline cylindrical shell considered the geometrical constraints ( $L / R$ and $R / t$ ratios) required to feature multiple mode transitions when placed under uniform axial compression and the size and tolerance constraints of the available 3D printer. The baseline cylinder had a length of $100 \mathrm{~mm}$, a radius of $40 \mathrm{~mm}$ and a thickness of $0.5 \mathrm{~mm}$. Stiffened/thicker ring 
regions of $10 \mathrm{~mm}$ were built on each end of the cylindrical shell to avoid damage from localized buckling at the edges. The reinforced region had a step-wise geometry to transition

121 from the edge to the center part of the cylindrical shell (i.e., effective length region). The

122 effective length $\left(L_{e f f}\right)$ of the central part of the shell was thus $80 \mathrm{~mm}$. The cylinder had an $L_{e f f} / R$ ratio of 2 and an $R / t$ ratio of 80 . The printing orientation and fill pattern have an 124 important effect on the mechanical properties of the printed part [29]. Based on tensile tests of 125 PLA printed coupons (Type IV as per ASTM D638 standard) the elastic modulus in the radial and vertical directions of the shells was 3.05 GPa and 2.57 GPa, respectively.

All specimens used the baseline dimension mentioned above, yet they featured variations depending on the design class. The SGI cylinders were seeded from a single mode with an amplitude of $200 \%$ of the total shell thickness. The shell thickness of the baseline uniform

130 cylinder was $0.5 \mathrm{~mm}$, which leads to a $1 \mathrm{~mm}$ maximum amplitude variation to the mid-plane

131 of the shell. The NSD cylinders used $0.5 \mathrm{~mm}$ thickening patterned patches, which increased

132 the total thickness of certain regions to $1 \mathrm{~mm}$. The LCI cylinder consisted of an assembly of 133 two concentric uniform cylinders with $0.5 \mathrm{~mm}$ thick walls separated by a $5 \mathrm{~mm}$ gap; where 134 the outside cylinder had an inner diameter of $80 \mathrm{~mm}$ and the inside one had an inner diameter 135 of $70 \mathrm{~mm}$.

136 The geometry models were built in using SolidWorks [30] and then converted into a *.stl 137 type file for 3D printing purposes. All shells were fabricated through 3D polymer-based 138 printing (MakerBot Replicator 2, MakerBot Industries, New York City) using a PLA 139 (Polylactic acid) filament. The printer heats the PLA filament and extrudes it through a nozzle.

140 The filament cools rapidly once it's placed on the building plate and the process is repeated to 141 make a solid object in a layer by layer manner as shown in Fig. 2(a).

142 All cylinders were tested under uniform axial compression in a universal testing frame as 143 shown in Fig. 2(b). The test setup made use of top and bottom loading fixtures with a 
144 pressure-tight fit to the test cylinder ends. Loading fixtures were fabricated using a ceramic-

145 based 3D printer (ZPrinter 250, 3D Systems, Rock Hill). The fixtures helped avoid damage to

146 the cylinder edges, provided a uniform edge loading pressure and created a condition of fixity

147 at the cylinder edges. The bottom loading fixture was a $20 \mathrm{~mm}$ thick circular plate with a

148 radius of $53 \mathrm{~mm}$, and provided with a circular (40.25 mm average diameter) groove (10 mm

149 deep and $4 \mathrm{~mm}$ wide) to allow connection with the test unit. The bottom loading fixture was

150 supported by a rigid surface while loading was applied through a spherical bearing placed at

151 the center of the top fixture. The top fixture had the same features as the bottom one but it was

152 also provided with a $20 \mathrm{~mm}$ cone in the center to uniformly distribute the load from a metallic

153 sphere attached to the universal testing frame. All cylinders were subjected to axial

154 compression under displacement control to a total shortening of $1.0 \mathrm{~mm}$ and then unloaded.

155 The load and displacement records obtained from the load cell and displacement transducer of

156 the universal testing frame were the only measurements taken.

a

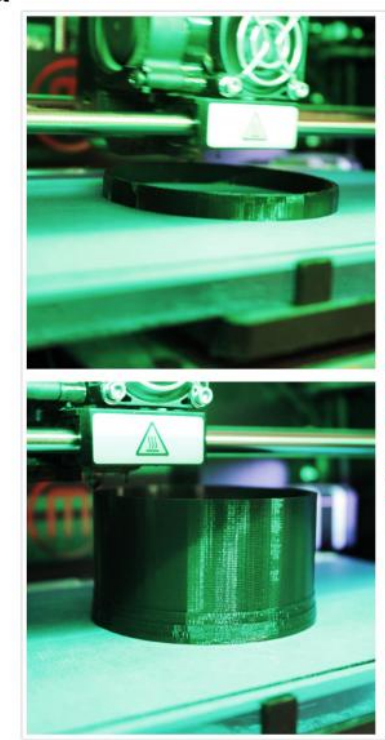

157

158

159 b
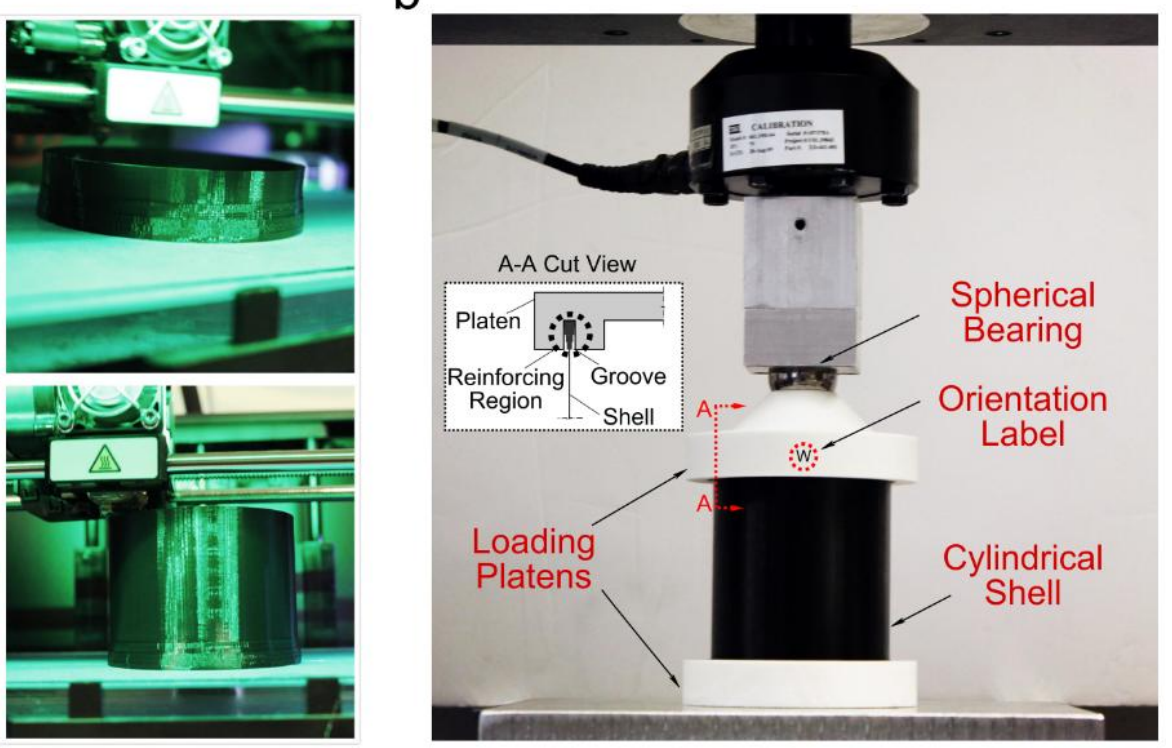

Figure 2. Fabrication and test of a cylindrical shell:

(a) Representative images of the 3D printing process; (b) Test setup for axial compression. 


\section{Test Results}

161

162

163

164

165

166

167

168

171

172

173

174

175

176

177

\subsection{SGI CONCEPT}

The rationale behind the SGI design concept is to provide the cylinder with a governing seeded imperfection over other random imperfections on the shell surface such that the postbuckling response can be tailored. Low buckling modes are usually used to assess postbuckling behavior with the aim of estimating critial load. In this study, higher-order modes were considered in order to provide more geometric options for seeded imperfection designs. Possible mode shapes were predicted for the baseline uniform cylinder using ABAQUS [31] as shown in Fig. 3(a). Periodic buckling modes and their locus can be interpreted along a half circle known as the Koiter circle [32] in terms of a dimensionless axial and circumferential wavenumber. It can be seen that the first 100 modes are along the perimeter of the half circle while the next 100 modes are slightly offset from the perimeter.

a

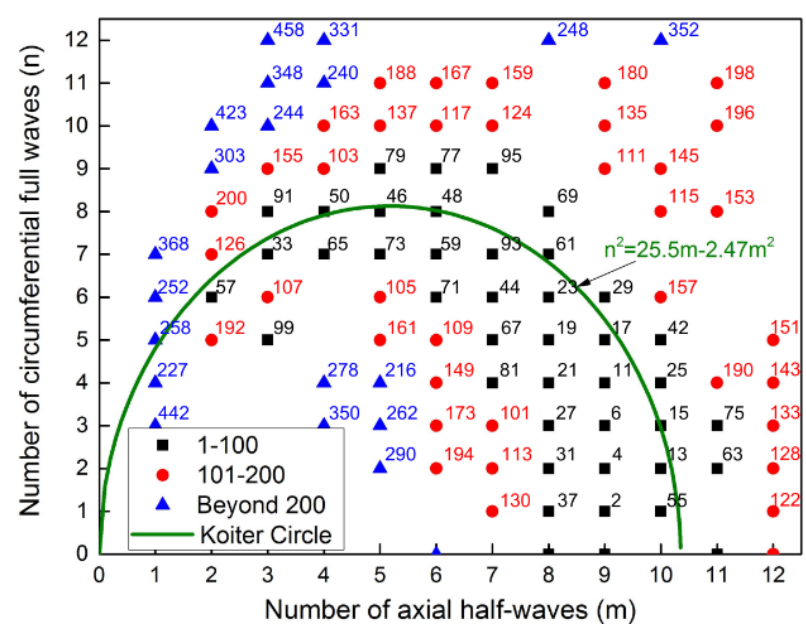

b

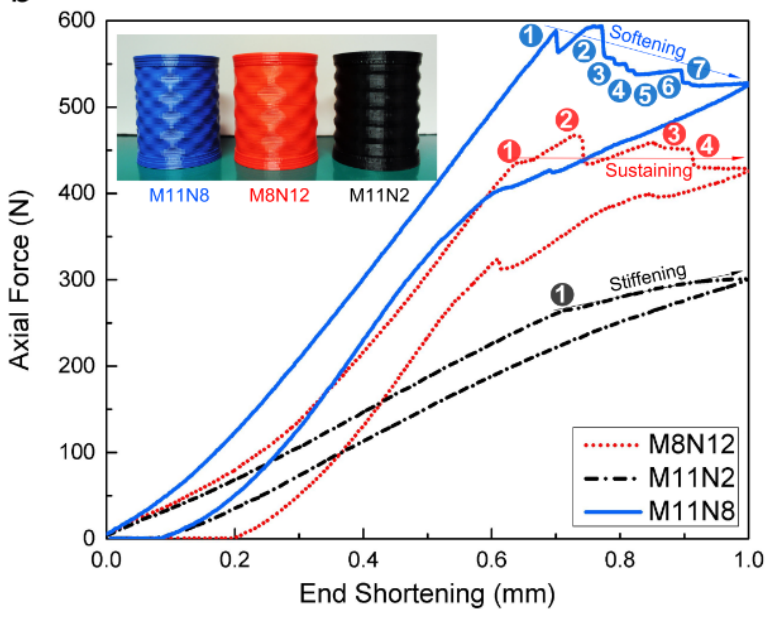

Figure 3. SGI design concept:

(a) Mode shapes as design variables; (b) Obtained postbuckling response of baseline designs.

Three prototype SGI cylinders, each seeded from a single mode shape on the Koiter circle were fabricated as shown in the inserts of Fig. 3(b). In the test unit naming convention "M" represents the half wavenumber in the axial direction while " $\mathrm{N}$ " indicates the circumferential 
178 full wavenumber. Thus, the SGI cylinders featured a different initial geometry but had an equal maximum amplitude variation of $1 \mathrm{~mm}$ with respect to the cylinder's mid-plane.

180 The resulting responses of axial load vs. end shortening for the SGI cylinders are given in

181 Fig. 3(b). It can be seen that the applied axial deformation and the initial stiffness were

182 recovered upon unloading in all tests. As expected, there is significant response variation

183 between the test results under exactly same test setup and loading sequence. Due to the 184 different number of waves on two directions, the initial stiffness and maximum load of the 185 SGI cylinders is different. It can be observed that cylinder M11N8 had more localized 186 buckling events (seven), or mode transitions, than the other two SGI cylinders. Cylinder 187 M8N12 had four load drops in the response curve and cylinder M11N2 had only one small 188 load drop. The occurrence of buckling events usually started with a localized inward buckling 189 wave and future events generated along the circumferential direction, affecting neighboring 190 regions first. This is the reason why only one buckling event was observed for cylinder 191 M11N2, because the localized buckling events are difficult to propagate with only two waves 192 in the circumferential direction of the seeded imperfection design. A previous study by the 193 authors [23] showed that multiple mode jumps in the postbuckling regime were more likely to 194 occur when the seeding geometry had a relatively larger number of waves in the 195 circumferential direction. Compared to the magnitude of a single load drop, it is interesting to 196 see that $\Delta P_{\max }$ in cylinder M11N8 $(37.6 \mathrm{~N})$ is similar to that of cylinder M8N12 (33.3 N), 197 although they had a different number of mode transitions. The larger load drop is usually 198 associated with a larger localized buckling region. In contrast, $\Delta P_{\max }$ in cylinder M11N2 was 199 only $1.6 \mathrm{~N}$. In terms of the enclosed area $(A)$ in the force-deformation response curve, the 200 results in Fig. 3(b) show that the initial stiffness has a strong correlation with $\Delta P_{\max }$ and $A$; 201 where more dissipated energy is usually associated with a higher initial stiffness and a larger 202 maximum load. 
Even though only three SGI cylinders are shown here, the most interesting finding from these pilot tests is that seeded geometry plays an important role in modifying the occurrence of buckling events. The three SGI cylinders showed quite different postbuckling responses under the same seeding amplitude, including softening (M11N8), sustaining (M8N12) and stiffening (M11N2) responses. Different M-N combinations can thus be used to generate a variety of seeding geometries such that the postbuckling behaviour can be tailored. Overall, a targeted postbuckling behavior can be achieved if a seeded initial shape is properly selected.

\subsection{NSD CONCEPT}

The rationale for NSD designs is to trigger mode transitions in the non-thickened regions such that the occurrences of localized buckling events are predictable. Two NSD cylinders were fabricated (see inserts in Fig. 4(a)) with different features along the shell surface.

Cylinder NSD-A contained "isolated" flexible regions, while the thickened regions of cylinder NSD-B were arranged in clustered patterns with well-connected flexible regions along the circumferential direction. The shell surface was uniformly discretized into 192 cells, or regions ( 8 in the axial direction by 24 in the circumferential direction). Both designs were symmetric and were provided with a basic pattern, as can be seen in the one-eighth model view of the insert in Fig. 4(a). Within the one-eighth domain, 8 out of the 24 cells were thickened to $1 \mathrm{~mm}$, which led to a thickening ratio of $1 / 3$ for both designs.

The resulting force-displacement responses of the NSD cylinders are shown in Fig. 4(a). The nonlinear deformations of the NSD cylinders, like that of the SGI cylinders, were recovered upon unloading. It can be seen that both NSD cylinders had similar initial stiffness due to the same thickening ratio (1/3). However, the layout of the patches plays an important role on the postbuckling behavior after the first buckling event. Local buckling of cylinder NSD-A started in the flexible region in the middle and later triggered other events in the 227 flexible regions at the bottom (see shaded regions on the postbuckling shape insert in Fig. 
4(a)). However, local buckling in this design had difficulty propagating along the 229 circumferential direction and buckling events were isolated between flexible regions. 230 Conversely, the localized buckling events of cylinder NSD-B propagated along the 231 circumferential direction due to the continuity among the flexible regions.
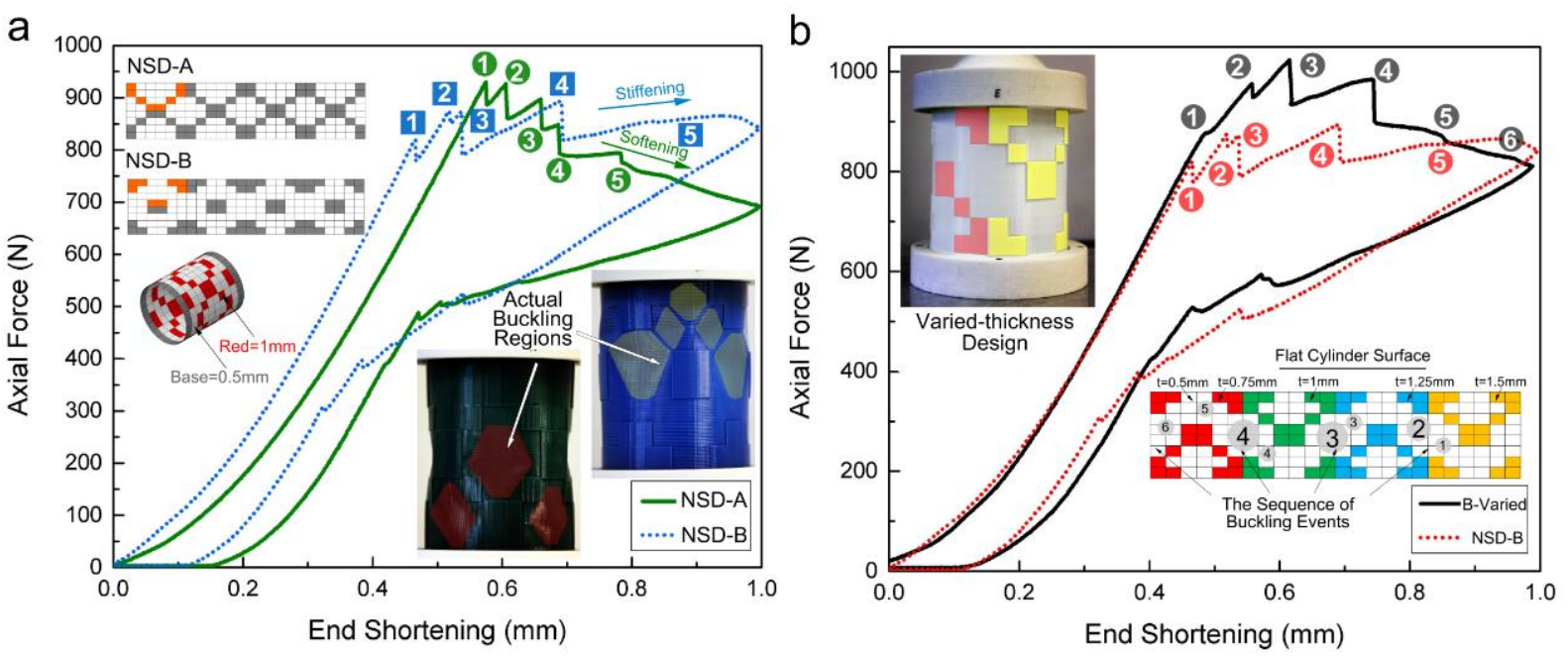

Figure 4. Obtained postbuckling responses of NSD cylinders:

(a) Two designs with different pattern layouts; (b) Effect of using non-uniform thickening regions throughout the shell surface.

NSD-A decreased rapidly after each critical event and the end stiffness was nearly negative, while the stiffness of cylinder NSD-B reduced gradually and a residual stiffness was still observed at the end of the loading process. In other words, NSD cylinders with "isolated" regions have a significant total load drop in the response curve and the first buckling load is usually the maximum critical load. On the other hand, a sustaining-type post-buckling response was observed in the NSD cylinder with "connected" flexible regions.

An additional cylinder was designed based on the baseline cylinder NSD-B, as shown in 244 the insert of Fig. 4(b). The patches in this cylinder varied in thickness along the 245 circumferential direction of the shell surface for each quarter segment, with values ranging 246 from $0.75 \mathrm{~mm}$ to $1.5 \mathrm{~mm}$ in increments of $0.25 \mathrm{~mm}$. This test showed that the buckling events 247 started near the middle $1.5 \mathrm{~mm}$ thick patches and then multiple mode transitions propagated 
along a path following regions with reduced stiffness patches. Thus, this test confirmed the

249 possibility of controlling not only the location of the buckling events but also the sequence of these events. Overall, a targeted postbuckling behavior can be achieved if discrete patterned patches, which generate non-uniform stiffness distributions, are properly placed.

\subsection{LCI CONCEPT}

The rationale behind the LCI concept is to take advantage of the collective response and interactions of an array of cylinders such that the postbuckling response of the system can be further modified and tailored. This concept is inspired by studies on the buckling of constrained columns [33, 34] and plates [35], as well as a pilot study by the authors [23]. The force-displacement responses for the double-walled LCI cylinder and a single uniform cylinder with a thickness of $0.5 \mathrm{~mm}$ are shown in Fig. 5(a). It can be observed from this plot that the force-deformation response for the LCI cylinder featured a significant increase in stiffness, a higher load capacity to the first buckling event, a larger value for the maximum load drop, and an increased amount of dissipated energy. It is also of value to note that the number of mode transitions in the LCI cylinder was twice the number generated in the uniform cylinder when subjected to an equal total end shortening.

The results of Fig. 5 (a)) seem to indicate that there was no interaction between walls. Thus, a numerical simulation was conducted out on a double-walled SGI cylinder group to showcase the interaction between each SGI component. Two SGI designs were selected with different m-n combinations, one as M8N8 and the other as M4N10. Both cylinders had the same length and wall thickness, and a gap of $5 \mathrm{~mm}$ was defined by making the inside cylinder (M8N8) have a smaller diameter than the outside one (M4N10). The simulated forcedeformation responses for the individual cylinders and the assembly (LCI design) for two 271 different gap sizes (2 and $5 \mathrm{~mm}$ ) are shown in Fig. 5(b). It can be seen that the individual 272 responses of the cylinders have different characteristics: a large load drop for M4N10 versus 
two smaller drops for M8N8. As observed in the test, the simulated result for the LCI cylinder

274 with a $5 \mathrm{~mm}$ gap had a combined response from each SGI cylinder without much interaction

275 between the walls. The number of mode transition was the addition from that of each

276 cylinder. However, the total load capacity, initial stiffness and enclosed area of the response

277 curve are higher for the LCI cylinder than for either of the single cylinders. Based on the

278 noted prior study [23], reducing the gap between the cylinders is expected to increase the

279 number of mode transitions. It can be seen (Fig. 5(b)) that the LCI cylinder with a 2 mm gap

280 featured a larger number of mode transitions and a sustaining response type. Another distinct

281 feature of the LCI design with a $2 \mathrm{~mm}$ gap is that the stiffness of the postbuckling equilibrium

282 branches (individual loading paths between mode transitions) did not degrade as rapidly as for

283 the LCI cylinder with a $5 \mathrm{~mm}$ gap due to interaction between the cylinders.
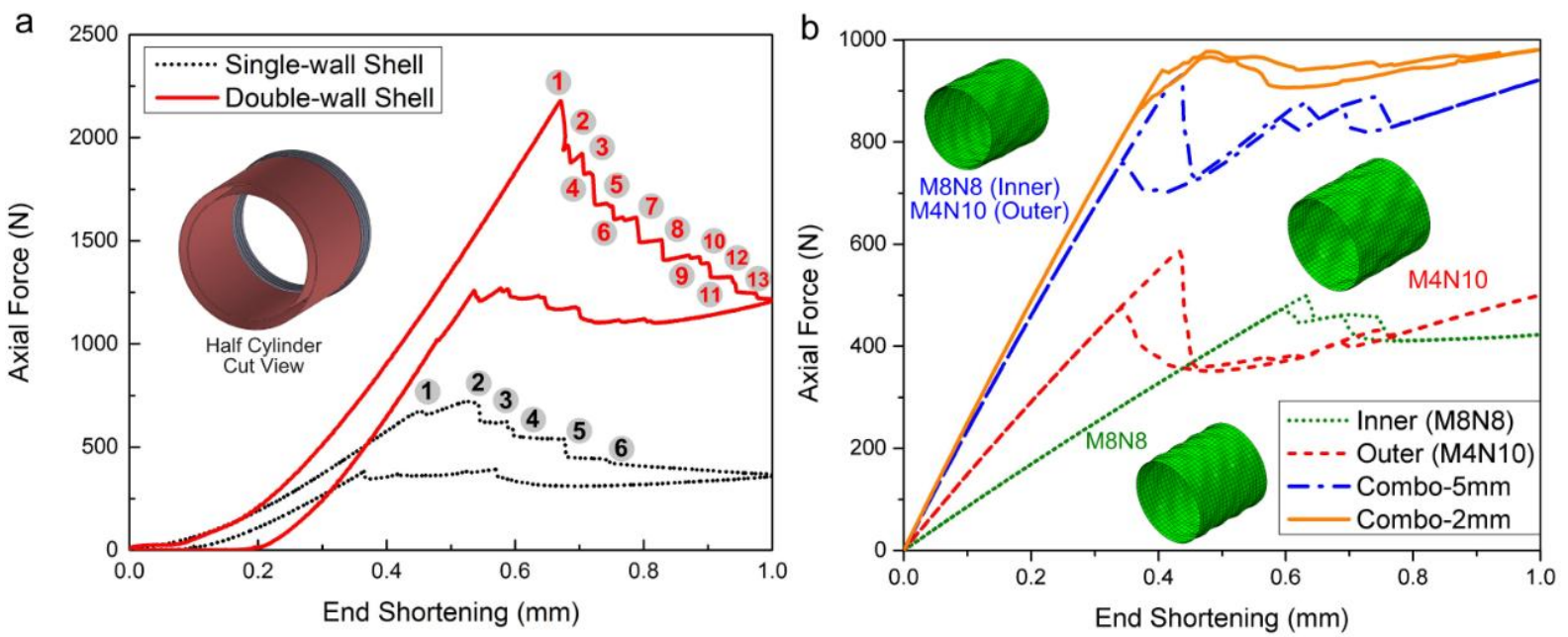

Figure 5. Postbuckling responses of double-walled LCI cylinders:

(a) Experiment results; (b) Numerical simulations.

\section{DISCUSSION}

As mentioned in the introduction, this research was motivated by the increased interest in using the instabilities of flexible systems for the development of smart materials and structures. Within this context, this study relates to the main research endeavor but focuses on exploring the use of cylindrical shells as a potential structural prototype. The second author 
and his colleagues have demonstrated that the attainment of a stable postbuckling response in

293 bilaterally constrained columns can be used to harvest energy for local use in devices such as

294 sensors without the need for external vibration as the energy source $[4,36]$, as conceptually

295 shown in Fig. 6(a). This is possible by using the kinetic energy released during the mode 296 transitions events in the postbuckling response to excite piezoelectric oscillators, which 297 transform the vibration energy caused by the sudden motion into power. Use of the 298 postbuckling response from cylinders is a clear extension but one that requires validation and characterization, particularly for optimized designs as the ones being pursued in this study.

a

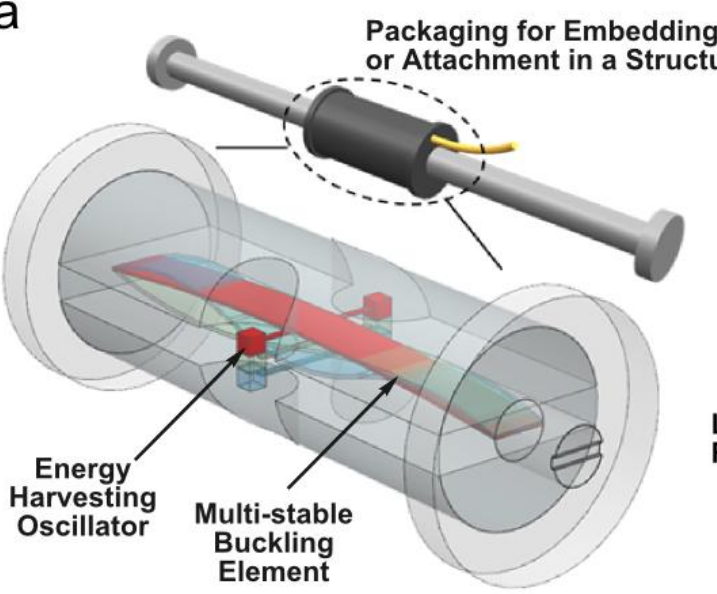

b

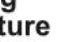
behaviour is scalable, which implies that such behaviour can have broad impact across scales. Such scale invariance for elastic postbuckling behavior has also been emphasized by other

Figure 6. Extrapolating findings to smart applications:

(a) Buckling-induced energy harvesting concept; (b) Miniaturization of cylinders with controllable postbuckling features.

Another aspect of interest for exploring uses is the miniaturization of the design concepts developed through this study. As shown in Fig. 6(b) the research endeavour featured in this communication started with relatively large cylinder prototypes $(\mathrm{L}=254 \mathrm{~mm}$ and $\mathrm{R}=101.5$ $\mathrm{mm}$ ) [23]. With the use of 3D printing, the scale of the baseline cylinder has been reduced by a factor of 100. Test data and simulation results indicate that the featured postbuckling studies [37] on cylinders made from a silicone-based elastomer. Finally, it should be noted 
that the PLA polymer suffered minor damage in the tests reported in this letter. Thus, further

313 development could benefit from the use of materials having the ability of shape change or 314 even shape memory.

315 Another potential use for mode transitions in the elastic postbuckling response of 316 cylinders includes the development of physical sensors. This is analogous to the way a jar cap 317 "pops" when opened, indicating the change in pressure. Thus, a sensor based on the 318 postbuckling response of cylindrical shells could be used to monitor pressure changes and 319 become activated only when certain (tailored) thresholds are met. This would mean that the 320 sensor does not need to be electronically wired and would remove the need for continuous 321 monitoring when only knowledge of the occurrence of the event is needed.

\section{Conclusions}

This letter showcased an alternative future for the postbuckling response of axiallycompressed cylindrical shells under the emerging trend of harnessing elastic instabilities for smart purposes. Three cylinder design concepts (SGI, NSD and LCI) that allow tailoring of their elastic postbuckling response were presented. Results showed that cylinders with seeded geometric imperfections (SGI) and non-uniform stiffness distributions (NSD) can attain a controllable postbuckling response due to the governing role of artificial imperfections

329 (geometry and stiffness). In both cases, the localized buckling events can be triggered in predefined regions; and careful selection of the seeded geometry and stiffness distribution can

331 lead to elastic postbuckling responses with tailorable features, which implies diverse design 332 opportunities. In addition, cylinders with internal lateral constraints and interactions (LCI) 333 allow the attainment of a relatively higher number of mode transitions in the elastic 334 postbuckling regime.

335 This research study presented new knowledge on the possibilities, extent and means to 336 control (and thus design) the elastic far postbuckling response of cylindrical shells. Full 
338 in the attainment and control of postbuckling response with desirable features can promote the

339 use of the presented cylindrical shell concepts for a variety of purposes of emerging interest;

340 including actuation, sensing, control, energy harvesting, energy dissipation, etc. Further, such

341 postbuckling response is expected to be scalable, reversible and robust.

\section{ACKNOWLEDGEMENTS}

The research described in this paper was carried out with partial funding from the U.S.

344 National Science Foundation under Grant number ECCS-1408506. The authors gratefully

345 acknowledge the technical support of Mr. Wassim Borchani and Mr. Brian Rook on the 346 experimental testing, and MSU's College of Engineering Division of Engineering and 347 Computing Services on specimen manufacturing, particularly to Messrs. Alex Hill, Anuj 348 Thaker and Jonathan Walby.

\section{REFERENCES}

[1] A.J. Crosby, Why should we care about buckling?, Soft Matter, 6 (2010) 5660-5660.

[2] R.L. Harne, K.W. Wang, A review of the recent research on vibration energy harvesting via bistable systems, Smart Mater Struct, 22 (2013) 023001.

[3] H. Kalathur, R.S. Lakes, Column dampers with negative stiffness: high damping at small amplitude, Smart Materials and Structures, 22 (2013) 084013.

[4] N. Lajnef, W. Borchani, R. Burgueno, S. Chakrabartty, Self-Powered Piezo-Floating-Gate Smart-Gauges Based on Quasi-Static Mechanical Energy Concentrators and Triggers, Sensors Journal, IEEE, 15 (2015) 676683.

[5] K. Seung-Won, K. Je-Sung, L. Jong-Gu, R. Junghyun, C. Maenghyo, C. Kyu-Jin, Flytrap-inspired robot using structurally integrated actuation based on bistability and a developable surface, Bioinspiration \& Biomimetics, 9 (2014) 036004.

[6] N. Lindahl, D. Midtvedt, J. Svensson, O.A. Nerushev, N. Lindvall, A. Isacsson, E.E.B. Campbell, Determination of the Bending Rigidity of Graphene via Electrostatic Actuation of Buckled Membranes, Nano Letters, 12 (2012) 3526-3531.

[7] D.P. Holmes, A.J. Crosby, Snapping Surfaces, Advanced Materials, 19 (2007) 3589-3593.

[8] W.M. Huang, H.B. Lu, Y. Zhao, Z. Ding, C.C. Wang, J.L. Zhang, L. Sun, J. Fu, X.Y. Gao, Instability/collapse of polymeric materials and their structures in stimulus-induced shape/surface morphology switching, Mater Design, 59 (2014) 176-192.

[9] W. Shan, Z. Chen, Mechanical instability of thin elastic rods, Journal of Postdoctoral Research February, 1 (2013) 8.

[10] V.G.A. Goss, G.H.M. Heijden, J.M.T. Thompson, S. Neukirch, Experiments on snap buckling, hysteresis and loop formation in twisted rods, Exp Mech, 45 (2005) 101-111.

[11] D. Bigoni, F. Bosi, F. Dal Corso, D. Misseroni, Instability of a penetrating blade, Journal of the Mechanics and Physics of Solids, 64 (2014) 411-425.

[12] A. Pandey, D.E. Moulton, D. Vella, D.P. Holmes, Dynamics of snapping beams and jumping poppers, EPL (Europhysics Letters), 105 (2014) 24001. 
[13] J. Huang, J. Liu, B. Kroll, K. Bertoldi, D.R. Clarke, Spontaneous and deterministic three-dimensional curling of pre-strained elastomeric bi-strips, Soft Matter, 8 (2012) 6291-6300.

[14] P.-O. Mouthuy, M. Coulombier, T. Pardoen, J.-P. Raskin, A.M. Jonas, Overcurvature describes the buckling and folding of rings from curved origami to foldable tents, Nat Commun, 3 (2012) 1290. [15] Y. Klein, E. Efrati, E. Sharon, Shaping of Elastic Sheets by Prescription of Non-Euclidean Metrics, Science, 315 (2007) 1116-1120.

[16] C.D. Santangelo, Buckling thin disks and ribbons with non-Euclidean metrics, EPL (Europhysics Letters), 86 (2009) 34003.

[17] Z.W. Yao, M. Bowick, X. Ma, R. Sknepnek, Planar sheets meet negative-curvature liquid interfaces, EPL (Europhysics Letters), 101 (2013) 44007.

[18] M. Dias, A. , C.D. Santangelo, The shape and mechanics of curved-fold origami structures, EPL (Europhysics Letters), 100 (2012) 54005.

[19] Q. Guo, A.K. Mehta, M.A. Grover, W. Chen, D.G. Lynn, Z. Chen, Shape selection and multi-stability in helical ribbons, Applied Physics Letters, 104 (2014) 211901.

[20] G.W. Hunt, I. Ario, Twist buckling and the foldable cylinder: an exercise in origami, Int J Nonlin Mech, 40 (2005) 833-843.

[21] J. Shim, C. Perdigou, E.R. Chen, K. Bertoldi, P.M. Reis, Buckling-induced encapsulation of structured elastic shells under pressure, Proceedings of the National Academy of Sciences, 109 (2012) 5978-5983.

[22] S. Knoche, J. Kierfeld, Secondary polygonal instability of buckled spherical shells, EPL (Europhysics Letters), 106 (2014) 24004.

[23] R. Burgueño, N. Hu, A. Heeringa, N. Lajnef, Tailoring the elastic postbuckling response of thin-walled cylindrical composite shells under axial compression, Thin Wall Struct, 84 (2014) 14-25.

[24] R.V. Southwell, On the General Theory of Elastic Stability, Philosophical Transactions of the Royal Society of London. Series A, Containing Papers of a Mathematical or Physical Character, 213 (1914) 187-244.

[25] L.H. Donnell, A new theory for the buckling of thin cylinders under axial compression and bending, Trans. Asme, 56 (1934) 795-806.

[26] T. von Karman, H.-S. Tsien, The Buckling of Thin Cylindrical Shells under Axial Compression, Journal of Aeronautical Sciences, 8 (1941) 303-312.

[27] H.F. Michielsen, The Behavior of Thin Cylindrical Shells After Buckling Under Axial Compression, Journal of the Aeronautical Sciences (Institute of the Aeronautical Sciences), 15 (1948) 738-744.

[28] C.R. Calladine, Theory of shell structures, Cambridge University Press, 1989.

[29] C. Jiang, G.F. Zhao, A Preliminary Study of 3D Printing on Rock Mechanics, Rock Mech Rock Eng, (2014) $1-10$.

[30] Dassault Systèmes, SolidWorks 2013 x64 Edition, in, Waltham, Massachusetts, USA, 2013.

[31] Dassault Systèmes, ABAQUS, User Manual Version 6.12, 2012.

[32] G. Hunt, G.J. Lord, M.A. Peletier, Cylindrical shell buckling: a characterization of localization and periodicity, Discrete and Continuous Dynamical Systems Series B, 3 (2003) 505-518.

[33] H. Chai, The post-buckling response of a bi-laterally constrained column, J Mech Phys Solids, 46 (1998) $1155-1181$.

[34] P. Holmes, G. Domokos, J. Schmitt, I. Szeberényi, Constrained Euler buckling: an interplay of computation and analysis, Comput Method Appl M, 170 (1999) 175-207.

[35] H. Chai, On the post-buckling behavior of bilaterally constrained plates, Int J Solids Struct, 39 (2002) 29112926.

[36] N. Lajnef, R. Burgueño, W. Borchani, Y. Sun, A concept for energy harvesting from quasi-static structural deformations through axially loaded bilaterally constrained columns with multiple bifurcation points, Smart Mater Struct, 23 (2014) 055005.

[37] A. Lazarus, P.M. Reis, Soft Actuation of Structured Cylinders through Auxetic Behavior, Advanced Engineering Materials, (2015) doi: 10.1002/adem.201400433. 